\title{
Metastatic prostate carcinoma detected at lymph node dissection for thyroid papillary carcinoma: report of a case
}

\author{
Takayuki Yamamoto $\cdot$ Yoshihiro Tominaga • \\ Toyonori Tsuzuki · Takahisa Hiramitsu • \\ Koji Nanmoku
}

Received: 15 August 2011/Accepted: 17 October 2011/Published online: 20 December 2011

(C) The Japan Society of Clinical Oncology 2011

\begin{abstract}
Skip metastasis from prostate cancer to cervical lymph nodes is very uncommon. We report the case of a 74-year-old man who was admitted to our hospital because of a thyroid cancer. Preoperative computed tomography (CT) imaging of the head and neck verified the previously detected mass at the left lobe of the thyroid gland. Cervical lymph nodes or signs of lung metastasis could not be found. The patient subsequently underwent total thyroidectomy with dissection of the central (I, II, III) and lateral zone lymph nodes (IV, Va, VI, VII). The diagnosis became T2ExONOM0. Histology verified a differentiated papillary thyroid carcinoma. Immunohistological examination showed that the origin of the metastases in the left cervical lymph nodes was the prostate and not the papillary thyroid carcinoma (PSA, PAP, p501S, p504S positive, CK7, CK20, p63, thyroglobulin-negative). The patient was referred to a urologist and prostate carcinoma was diagnosed by biopsy. The PSA level was $56.87 \mathrm{ng} / \mathrm{ml}$ and transrectal ultrasonography showed a prostate mass of $46 \times 40 \times 55 \mathrm{~mm}$ in size. Hormonal therapy decreased the PSA level to $3.74 \mathrm{ng} / \mathrm{ml}$ at 6 months after the operation. His general health condition is now good. This is the first report, as far as we know, describing a man whose prostate cancer was
\end{abstract}

This case was presented at the 23rd Annual Congress of the Japan Association of Endocrine Surgeons (7 July 2011, Tokyo).

T. Yamamoto $(\bowtie) \cdot$ Y. Tominaga · T. Hiramitsu $\cdot$ K. Nanmoku Department of Transplant and Endocrine Surgery,

Nagoya Daini Red Cross Hospital, 2-9 Myouken chou,

Shouwa-ku, Nagoya, Japan

e-mail: tyamamoto1976@yahoo.co.jp

T. Tsuzuki

Department of Pathology, Nagoya Daini Red Cross Hospital,

Nagoya, Japan detected in the cervical lymph nodes after undergoing surgery for thyroid cancer.

Keywords Prostate carcinoma - Thyroid carcinoma . Left cervical lymph node

\section{Introduction}

Sometimes a cancer will be detected by the presence of lymph node metastasis. The diagnosis of the original lesion is often difficult using routine histology, but immunohistological staining can demonstrate a cell function and differentiation that leads to a precision of the pathological diagnosis [1].

Cervical lymph nodes are common sites of metastases for head and neck cancers. However, cancers that originate from other sites can metastasize to the cervical lymph nodes, but metastasis from prostate cancer is an extremely rare origin. We report a case of prostate carcinoma metastasizing to the cervical lymph nodes and detected in connection with an operation for papillary thyroid carcinoma.

\section{Case report}

A 74-year-old man presented with complaints of left cervical and clavicular pain of 6 months' duration. He underwent neck computed tomography (CT) at a nearby clinic. A mass the size of a quail egg was detected at the left lobe of the thyroid gland. Fine needle aspiration cytology (FNAC) showed papillary carcinoma. The patient was referred to our hospital. Clinical examination revealed an elastic, hard, mobile, palpable, and painful mass the size 
of a chicken egg in the left lobe of the thyroid gland. Chest $\mathrm{X}$-ray, blood count, and serum biochemistry were normal. Thyroid-stimulating hormone (TSH), free triiodothyronine (FT3), and free thyroxine (FT4) were $1.07 \mathrm{IU} / \mathrm{ml}, 2.55 \mathrm{ng} /$ $\mathrm{dl}$, and $1.01 \mathrm{ng} / \mathrm{dl}$, respectively, all within normal range. Thyroglobulin, calcitonin, carcinoembryonic antigen (CEA), and antithyroglobulin antibody were normal. Preoperative CT imaging of the head and neck verified the previously detected mass at the left lobe of the thyroid gland measuring $31 \times 28 \mathrm{~mm}$. The surface was slightly irregular, but the border recognized. Cervical lymph nodes or signs of lung metastasis could not be found.

The patient subsequently underwent total thyroidectomy with dissection of the central (I, II, III) and lateral zone lymph nodes (IV, Va, VI, VII). The diagnosis became T2ExONOM0. The vagus and recurrent laryngeal nerves, the left phrenic and accessory nerves were preserved with a nerve stimulator monitoring (NIM) during the operation. The lower left parathyroid gland was removed together with the thyroid gland, but the parathyroid was autografted in the right sternocleidomastoid muscle. The other three parathyroid glands were preserved in situ. The postoperative course was uneventful.

The thyroid tumor was $34 \times 26 \times 28 \mathrm{~mm}$ in size and histopathologically a well-differentiated papillary carcinoma was diagnosed. Specimens representing the right central zone lymph nodes (I, II, III) showed no malignancy. Of the lymph nodes from the lateral zone (IV, Va, VI, VII) 4 of 17 showed growth of adenocarcinoma (Fig. 1). The case was considered to be pT3pEx1pN0pstageIII by pathological examination. Immunohistochemically, the neoplastic cells in the lymph nodes were positive for PSA,

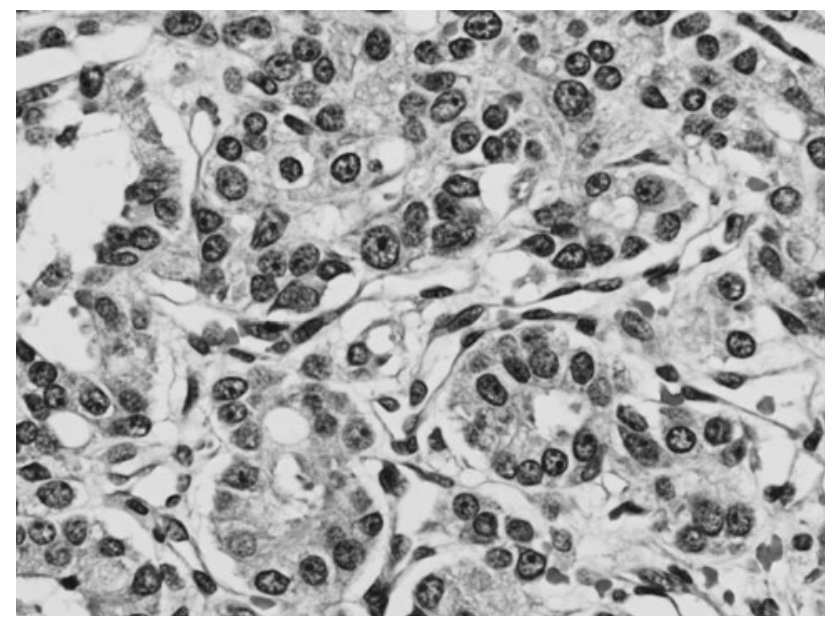

Fig. 1 Prostate cancer metastasizing to cervical lymph nodes. The cells have macronucleoli and clear or vacuolated cytoplasm. There were no cytologic findings that support the diagnosis of papillary thyroid carcinoma such as grooves, inclusions, and cytoplasmic details

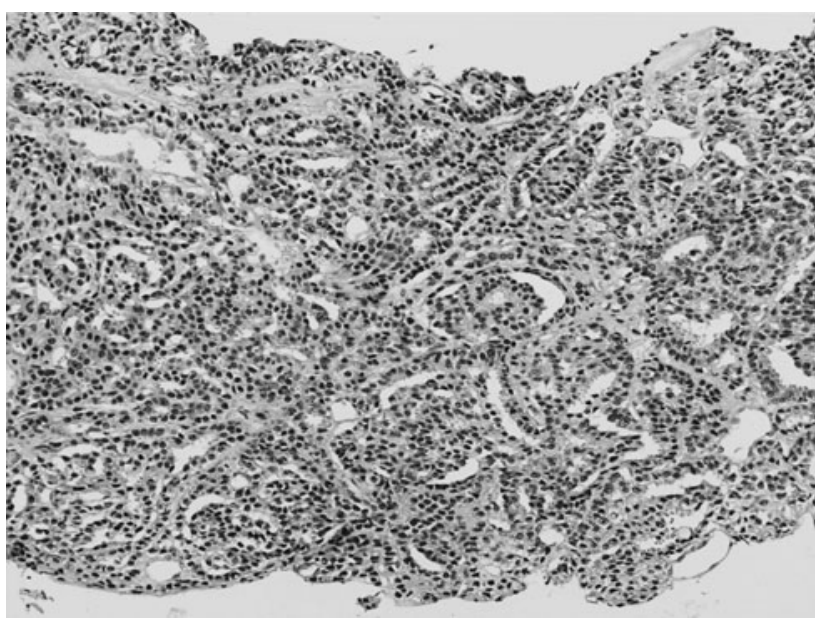

Fig. 2 Primary prostate cancer (adenocarcinoma)

PSAP, p501S, p504S, and androgen receptor but were uniformly negative for CK7, CK20, p63, and thyroglobulin. The morphological and immunohistochemical findings were consistent with an adenocarcinoma of prostate origin. Urologic consultation was obtained. The PSA level was $56.87 \mathrm{ng} / \mathrm{ml}$ and transrectal ultrasonography showed a prostate mass of $46 \times 40 \times 55 \mathrm{~mm}$ in size. Biopsy showed poorly differentiated prostate cancer with Gleason scores $4+5=9$ (Fig. 2). Bone metastasis was not found using whole body bone scintigraphy. The patient was given hormonal therapy after undergoing an otherwise negative metastatic work-up. Hormonal therapy decreased the PSA level to $3.74 \mathrm{ng} / \mathrm{ml}$ at 6 months after the operation. His general health condition is now good.

\section{Discussion}

Prostate cancer frequently metastasizes to regional lymph nodes. In a series consisting of 753 postmortem examinations, regional lymph node metastases were shown in $63 \%$ of the cases. The metastases were found both in pelvic and para-aortic lymph nodes; sometimes only the para-aortic region was involved [2]. Distant metastases to supradiaphragmatic lymph nodes were rare, and occurred usually in the left supraclavicular region. However, this was seen only in patients with widespread metastases [3-6]. In our case, undergoing total thyroidectomy and regional lymph node dissection for thyroid papillary cancer, the lymph node metastases did not contain growth of thyroid carcinoma but represented adenocarcinoma originating from the prostate. That was a surprising finding and it must be extremely rare that cervical lymph node metastasis originating from prostate cancer can be accidentally detected by pathological examination after thyroid surgery. 
The most common clinical symptoms of primary prostate cancer are dysuria, urinary retention, hematuria, and incontinence. Occasionally, symptoms secondary to a metastatic disease are the presenting complaint. The presence of prostate cancer metastases indicates a poor prognosis, because the detection of one lesion suggests multiple undetected lesions. Metastatic prostate cancer remains an incurable disease, and that a detection of prostate cancer can occur by neck dissection for head and neck cancer must be an oncologically rare finding [4].

Spread of prostatic adenocarcinoma occurs most commonly through direct or perineural growth into the pelvic viscera or vertebral bodies. Lymphatic spread is often seen in the obturator-hypogastric lymph nodes. Metastases to the head and neck region are postulated to be spread via the vertebral venous system or Batson's plexus, accessible via direct growth from the primary cancer site [7-10]. Cancer metastases in the cervical chain from distant, non-head and neck primary sites are rare. Of these non-head and neck primary cancers, lung, kidney, and breast cancers are most frequently encountered as metastasizing to the cervical chain. Despite the high prevalence of prostate cancer, metastases to the cervical lymphatic chains are uncommon. In an extensive review of urogenital cancer metastases, Flocks and Boatman [10] found only $0.4 \%$ of prostate cancer ( 6 of 1,500 cases) metastasizing to cervical nodes. In a retrospective review of urogenital metastases to the head and neck, Hessan et al. [11] found no cases ( 0 of 415 cases) of prostate cancer showing spread to the cervical nodes. There were few reports concerning hypogastric cancer with cervical lymph nodes except for prostate carcinoma. Keepanasseril et al. [12] reported a case that presented with metastatic cervical lymph nodes which is an extremely rare presentation of an ovarian carcinoma.

Positive immunohistological staining for thyroglobulin suggests a primary thyroid malignancy. However, as $20-30 \%$ of anaplastic carcinomas also stain positively for thyroglobulin, a negative result is not diagnostic [13]. Staining for immunoreactive thyroglobulin, if positive, is very helpful, but metastatic tumors may have an uptake of thyroglobulin from the surrounding gland [14]. In our case, thyroglobulin staining of the cervical lymph nodes was negative, whereas PSA staining was positive. PSA used as an immunohistochemical marker for prostate gland epithelium, offers a highly sensitive and specific examination for diagnosing metastatic prostate cancer [15].

Although cervical lymph node metastasis from a prostate cancer accompanying thyroid papillary carcinoma is extremely rare, surgeons should keep in mind the possibility of adenocarcinoma metastases, especially in patients with left cervical lymph nodes.

Conflict of interest The authors declare that they have no conflict of interest.

\section{References}

1. Koichi M, Shosuke M, Toshiaki S et al (1994) A clinicopathological analysis of cases with metastatic tumors to lymph node in special reference to cancer of unknown primary site. J Jpn Soc Cancer Ther 29:978-989

2. Saitoh H, Yoshida K, Uchijima Y et al (1990) Two different lymph node metastatic patterns of a prostatic cancer. Cancer 65:1843-1846

3. Wang HJ, Chiang PH, Peng JP et al (2004) Presentation of prostate carcinoma with cervical lymphadenopathy: report of three cases. Med J 27:840-844

4. Copeland B, Clark JM, Sura A et al (2001) Prostate carcinoma metastatic to the cervical lymph nodes: report of two cases and review of the literature. Am J Otolaryngol 22:420-423

5. Ozgur A, Ilker Y, Turkeri LN (2003) Cervical lymph node enlargement on the right side as the initial manifestation of metastatic prostate cancer. Arch Esp Urol 56:859-861

6. Carleton J, van der Riet P, Dahm P (2005) Metastatic prostate cancer presenting as an asymptomatic neck mass. Prostate Cancer Prostatic Dis 8:293-295

7. Albers DD (1970) Adenocarcinoma of the prostate metastatic to the mandible. J Am Dental Assoc 81:883-886

8. Batson OV (1995) The function of the vertebral veins and their role in the spread of metastases. Clin Orthop Relat Res 312:4-9

9. McLaughlin AP, Saltzstein SL, McCullough DL et al (1976) Prostatic carcinoma: incidence and location of unsuspected lymphatic metastases. J Urol 115:89-94

10. Flocks RH, Boatman DL (1973) Incidence of head and neck metastases from genito-urinary neoplasms. Laryngoscope 83: $1527-1539$

11. Hessan H, Strauss M, Sharkey F (1986) Urogenital tract carcinoma metastatic to the head and neck. Laryngoscope 96:13521356

12. Keepanasseril A, Suri V, Gupta N, Ghoshal S (2008) Transitional cell carcinoma of the ovary: unusual presentation as metastatic cervical lymph node. J Obstet Gynaecol Res 34:696-698

13. Nakhjavani MK, Gharib H, Goellner JR et al (1997) Metastasis to the thyroid gland. Cancer 79:574-578

14. Livolsi VA, Montone K, Sack M (1999) Pathology of thyroid disease. In: Sternberg SS (ed) Diagnostic surgical pathology, 3rd edn. Lippincott Williams and Wilkins, Philadelphia, pp 529-587

15. Nash F, Melezinek I (2000) The role of prostate specific antigen measurement in the detection and management of prostate cancer. Endocr Relat Cancer 7:37-51 